PROCEEDINGS OF THE

AMERICAN MATHEMATICAL SOCIETY

Volume 138, Number 6, June 2010, Pages 2187-2194

S 0002-9939(10)10241-X

Article electronically published on February 1, 2010

\title{
MONOTONE BIVARIATE MARKOV KERNELS WITH SPECIFIED MARGINALS
}

\author{
MOTOYA MACHIDA AND ALEXANDER SHIBAKOV
}

(Communicated by Richard C. Bradley)

\begin{abstract}
Given two Markov kernels $k$ and $k^{\prime}$ on an ordered Polish space, such that $k$ is stochastically dominated by $k^{\prime}$, we establish the existence of: (i) a monotone bivariate Markov kernel whose marginals are $k$ and $k^{\prime}$ and (ii) an upward coupler from $k$ to $k^{\prime}$. This extends the results of Strassen, Kamae, Krengel and O'Brien to Markov kernels. Two examples are also given. The first is a simple illustration of our original motivation for this work, while the second demonstrates the optimality of our main result. The key technique is a combination of the standard probability/charge approach and the use of measurable selections of multivalued measurable maps.
\end{abstract}

\section{INTRODUCTION}

In his well-known paper [10, Strassen studied the existence of a probability measure $\lambda$ with given marginals $p$ and $p^{\prime}$. His results played a central part in the work of Kamae, Krengel and O'Brien [5] who carried out an extensive investigation of ordered pairs of distributions defined on ordered Polish spaces in the sense of Nachbin [7. In the present paper, the authors extend the results of Strassen, Kamae, Krengel and O'Brien to pairs of Markov kernels on ordered Polish spaces. The original motivation for our work stemmed from the desire to establish a general result guaranteeing the existence of an upward coupler that plays a key role in Fill's perfect rejection sampling algorithm [4. While the existence of such a coupler in the case of a countable probability space is an easy exercise, the case of an uncountable Polish space requires some care, as explained below.

Before we present any details, a few basic definitions are in order. A Markov kernel $k$ on a Polish (= complete separable metric) space $S$ is a real valued function on $S \times \mathcal{B}$ (here $\mathcal{B}$ is the Borel $\sigma$-algebra on $S$ ) with the following two properties: $k(r, \cdot)$ is a probability measure on the measurable space $(S, \mathcal{B})$ for each $r \in S$, and $k(\cdot, G)$ is a $\mathcal{B}$-measurable function for every $G \in \mathcal{B}$. The second condition can be weakened by considering open sets instead of measurable ones, giving an equivalent definition. A partial order on a Polish space $S$ is closed if it is closed as a subset of $S \times S$. Any such order naturally leads to an order on the set of all probability measures on $S$. Namely, a probability measure $p$ on $S$ is said to be stochastically

Received by the editors February 9, 2009, and, in revised form, October 5, 2009.

2010 Mathematics Subject Classification. Primary 60E15; Secondary 62E15, 60J05.

Key words and phrases. Probability measures with given marginals, stochastically monotone Markov kernel, measurable selection, coupling, Fill's algorithm.

(C)2010 American Mathematical Society Reverts to public domain 28 years from publication 
smaller than $p^{\prime}$ if $\int f d p \leq \int f d p^{\prime}$ for any monotone integrable $f$. In this case we write $p \preceq p^{\prime}$.

An easy corollary of Strassen's results is that $p \preceq p^{\prime}$ is a necessary and sufficient condition for the existence of a probability measure $\lambda$ on $\Delta=\left\{\left(s, s^{\prime}\right) \in S \times S\right.$ : $\left.s \leq s^{\prime}\right\}$ that satisfies

$$
p(\cdot)=\lambda\left(\pi_{1}^{-1}(\cdot)\right) \text { and } p^{\prime}(\cdot)=\lambda\left(\pi_{2}^{-1}(\cdot)\right),
$$

where $\pi_{1}\left(s, s^{\prime}\right)=s$ and $\pi_{2}\left(s, s^{\prime}\right)=s^{\prime}$ are the coordinatewise projections from $\Delta$ to $S$. Such $\lambda$ is said to have $p$ and $p^{\prime}$ as its marginals.

Developing this theme a bit further, one says that a Markov kernel $k$ is stochastically dominated by another Markov kernel $k^{\prime}$ if $k(r, \cdot) \preceq k^{\prime}\left(r^{\prime}, \cdot\right)$ whenever $r \leq r^{\prime}$.

Every kernel $k$, along with initial distribution $\nu_{0}$ on $S$, determines the distribution of a Markov chain trajectory (i.e., a "sample path") $X=\left(X_{0}, X_{1}, \ldots\right)$. Kamae, Krengel and O'Brien characterized the property of stochastic dominance by a "realization" for a pair of trajectories. If $\nu_{0} \preceq \nu_{0}^{\prime}$ holds for a pair of initial distributions and $k$ is dominated by $k^{\prime}$, then there exists a pair of Markov chain trajectories

$$
X=\left(X_{0}, X_{1}, \ldots\right) \leq X^{\prime}=\left(X_{0}^{\prime}, X_{1}^{\prime}, \ldots\right)
$$

determined by $k$ and $k^{\prime}$, respectively, which maintains the pairwise order $X_{n} \leq X_{n}^{\prime}$ for all $n \geq 0$ (Theorem 2 of [5]). This is essentially a special case of the NachbinStrassen theorem for the existence of a probability measure on $S^{\infty} \times S^{\infty}$ with given marginal distributions for $X$ and $X^{\prime}$.

As the example in Section 1.1 demonstrates, however, the "coupled" trajectory $\left(X_{n}, X_{n}^{\prime}\right)_{n=0,1, \ldots}$ obtained by this straightforward approach is not necessarily Markovian. The same example shows that the lack of the bivariate Markovian property is undesirable in certain applications.

A natural improvement of this technique would be to seek a kernel $K$ on $\Delta$ satisfying the marginal condition

$$
k(r, \cdot)=K\left(\left(r, r^{\prime}\right), \pi_{1}^{-1}(\cdot)\right) \text { and } k^{\prime}\left(r^{\prime}, \cdot\right)=K\left(\left(r, r^{\prime}\right), \pi_{2}^{-1}(\cdot)\right), \quad\left(r, r^{\prime}\right) \in \Delta .
$$

Such a kernel is easily seen to generate a Markov chain $\left(X, X^{\prime}\right)$ whose sample path satisfies (1.2). To construct such $K$ for a countable $S$, one can repeatedly apply the Nachbin-Strassen theorem to each pair $\left(r, r^{\prime}\right) \in \Delta$ to obtain a probability measure $K\left(\left(r, r^{\prime}\right), \cdot\right)$ satisfying (1.3). This brute-force construction of $K$ in the case of a general Polish $S$ might not necessarily result in a Markov kernel, however, as the measurability of $K(\cdot, E)$ is not guaranteed for all $\mathcal{B} \otimes \mathcal{B}$-measurable $E \subseteq \Delta$. The counterexample at the end of Section 2 can be modified to show the lack of bivariate measurability if $K$ is not selected carefully [e.g., for $k(r, \cdot) \equiv p(\cdot)$ and $k^{\prime}\left(r^{\prime}, \cdot\right) \equiv p^{\prime}(\cdot)$ as introduced in the same example].

This difficulty is resolved in Section 2 by the use of a measurable selection theorem (see Aubin and Frankowska [1]).

Finally, Section 3 of the paper deals with the existence of an upward coupler. A kernel $M$ is called upward if each probability measure $M(s, \cdot)$ is supported by $\left\{s^{\prime} \in S: s^{\prime} \geq s\right\}$. Again, the Nachbin-Strassen theorem implies that an upward $M$ satisfying $p^{\prime}(\cdot)=\int M(s, \cdot) p(d s)$ exists if and only if $p \preceq p^{\prime}$. Let $\mathcal{M}=\left\{M_{r, r^{\prime}}\right\}_{\left(r, r^{\prime}\right) \in \Delta}$ be a collection of upward kernels such that $M_{r, r^{\prime}}(s, E)$ is a measurable function of $\left(r, r^{\prime}, s\right)$ for every measurable set $E$. Then we call $\mathcal{M}$ an 
upward coupler if

$$
k^{\prime}\left(r^{\prime}, \cdot\right)=\int M_{r, r^{\prime}}(s, \cdot) k(r, d s) \quad \text { for every }\left(r, r^{\prime}\right) \in \Delta .
$$

In Section 3 we prove the existence of an upward coupler in the case when $k$ is stochastically dominated by $k^{\prime}$, which plays a key role in Fill's perfect rejection sampling algorithm (Section 7.2 of [4]). The following short argument will help to illustrate the importance of $\mathcal{M}$.

Fill's algorithm requires the upward coupler in order to construct the second trajectory coupled ex post facto with the first one. The first Markov chain trajectory $X$ starts alone from the initial distribution $\nu_{0}$ and evolves according to the Markov kernel $k$. Then, having started $X_{0}^{\prime}$ from $\nu_{0}^{\prime}$ in such a way that $X_{0} \leq X_{0}^{\prime}$, we can recursively generate $X_{n}^{\prime}$ according to the conditional distribution $M_{r, r^{\prime}}(s, \cdot)$ given $X_{n-1}=r, X_{n}=s$, and $X_{n-1}^{\prime}=r^{\prime}$. This realizes the second Markov chain $X^{\prime}$ satisfying (1.2).

1.1. An example. A kernel is called stochastically monotone if it stochastically dominates itself. Next we present two different constructions of coupled sample paths (1.2), whose marginals are both generated by the same stochastically monotone kernel $k$.

Consider an ordered Polish space $(S, \geq)$ where $S=[0,1]^{2}$ and $\geq$ is the usual (coordinatewise) partial order. Partition $S$ into $\{\mathbf{0}\}:=\{(0,0)\}$ (the minimum), $F_{1}:=(0,1 / 2]^{2}$ and $F_{2}:=S \backslash[0,1 / 2]^{2}$, and let $\delta_{s}(\cdot)$ denote the unit mass (i.e., the Dirac measure) at $s$. Finally, let $u_{i}(\cdot)$ be the uniform probability measure on $F_{i}$ [i.e., the normalized Lebesgue measure $\left.\mu(\cdot) / \mu\left(F_{i}\right)\right]$ for $i=1,2$. Define a stochastically monotone kernel

$$
k(s, d r)= \begin{cases}\frac{1}{3} \delta_{\mathbf{0}}(d r)+\frac{2}{3} u_{1}(d r) & \text { if } s=\mathbf{0} \\ \frac{1}{3} \delta_{\mathbf{0}}(d r)+\frac{2}{3} u_{2}(d r) & \text { if } s \in F_{1} \\ \frac{1}{3} u_{1}(d r)+\frac{2}{3} u_{2}(d r) & \text { if } s \in F_{2}\end{cases}
$$

which governs a move from $s$ to $r$. Next, construct a sample path $X=\left(X_{0}, X_{1}, X_{2}\right)$ starting from the stationary distribution

$$
\nu_{0}(\cdot)=\frac{1}{7} \delta_{\mathbf{0}}(\cdot)+\frac{2}{7} u_{1}(\cdot)+\frac{4}{7} u_{2}(\cdot)
$$

as well as $X^{\prime}=\left(X_{0}^{\prime}, X_{1}^{\prime}, X_{2}^{\prime}\right)$ starting at the maximum $\mathbf{1}=(1,1)$.

The space $S$ has the lumped states $\mathbf{0}, F_{1}$ and $F_{2}$, so an explicit construction of pairwise ordered sample paths is possible as we will show.

First, introduce a map $\zeta$ from $\{\mathbf{0}\} \cup F_{1}$ to $\{\mathbf{0}\} \cup F_{2}$ as follows:

$$
\zeta(s)= \begin{cases}\mathbf{0} & \text { if } s=\mathbf{0} \\ 2^{i} s & \text { if } s \in\left[0,2^{-i}\right]^{2} \backslash\left[0,2^{-i-1}\right]^{2} \text { for some } i \geq 1 .\end{cases}
$$

When $Z$ is distributed as $k(\mathbf{0}, \cdot), Z^{\prime}=\zeta(Z)$ is from $k\left(s^{\prime}, \cdot\right)$ with $s^{\prime} \in F_{1}$ and preserves the order $Z \leq Z^{\prime}$. Having sampled $\left(X_{0}, X_{1}, X_{2}\right)=\left(s_{0}, s_{1}, s_{2}\right)$ from $\nu_{0}\left(d s_{0}\right) k\left(s_{0}, d s_{1}\right) k\left(s_{1}, d s_{2}\right)$, we can then generate $\left(X_{1}^{\prime}, X_{2}^{\prime}\right)=\left(s_{1}^{\prime}, s_{2}^{\prime}\right)$ conditionally 
using

$$
\begin{cases}u_{1}\left(d s_{1}^{\prime}\right) \delta_{\zeta\left(X_{2}\right)}\left(d s_{2}^{\prime}\right) & \text { if } X_{0}=X_{1}=\mathbf{0} \\ u_{2}\left(d s_{1}^{\prime}\right) u_{1}\left(d s_{2}^{\prime}\right) & \text { if } X_{0} \in F_{1} \text { and } X_{1}=X_{2}=\mathbf{0} \\ u_{2}\left(d s_{1}^{\prime}\right) \delta_{X_{2}}\left(d s_{2}^{\prime}\right) & \text { if } X \in F_{1} \times\{\mathbf{0}\} \times F_{1} \\ \delta_{X_{1}}\left(d s_{1}^{\prime}\right) \delta_{\zeta\left(X_{2}\right)}\left(d s_{2}^{\prime}\right) & \text { if } X \in F_{1} \times F_{2} \times F_{1} .\end{cases}
$$

Otherwise, set $\left(X_{1}^{\prime}, X_{2}^{\prime}\right)=\left(X_{1}, X_{2}\right)$.

Clearly $\left(X_{1}, X_{2}\right) \leq\left(X_{1}^{\prime}, X_{2}^{\prime}\right)$, and the distribution of $\left(X_{1}^{\prime}, X_{2}^{\prime}\right)=\left(s_{1}^{\prime}, s_{2}^{\prime}\right)$ is determined as $k\left(\mathbf{1}, d s_{1}^{\prime}\right) k\left(s_{1}^{\prime}, d s_{2}^{\prime}\right)$. Therefore, $X^{\prime}$ is a Markov chain trajectory satisfying (1.2). When (1.2) is jointly Markovian, it has to be coalescing in the sense that $X_{n}=X_{n}^{\prime}$ for all $n \geq k$ once $X_{k}=X_{k}^{\prime}$ occurs. In the construction above, however, when $\left(X_{0}, X_{1}, X_{2}\right) \in F_{1} \times F_{2} \times F_{1}$ one can see that $X_{1}=X_{1}^{\prime}$ and $X_{2} \neq X_{2}^{\prime}$. Hence, the paired sample path $\left(X_{n}, X_{n}^{\prime}\right)_{n=0,1,2}$ cannot be jointly Markovian.

Now, let

$$
M_{r, r^{\prime}}\left(s, d s^{\prime}\right)= \begin{cases}\delta_{\zeta(s)}\left(d s^{\prime}\right) & \text { if }\left(r, r^{\prime}, s\right) \in\{\mathbf{0}\} \times\left(F_{1} \cup F_{2}\right) \times F_{1} ; \\ u_{1}\left(d s^{\prime}\right) & \text { if }\left(r, r^{\prime}, s\right) \in\left(\{\mathbf{0}\} \cup F_{1}\right) \times F_{2} \times\{\mathbf{0}\} ; \\ \delta_{s}\left(d s^{\prime}\right) & \text { otherwise }\end{cases}
$$

define an upward coupler $\mathcal{M}$ for $\left(r, r^{\prime}\right) \in \Delta$. Using $\mathcal{M}$ we can demonstrate how the construction via an upward coupler differs from the one above when they are used in Fill's algorithm. Construct the second trajectory $X^{\prime \prime}=\left(X_{0}^{\prime \prime}, X_{1}^{\prime \prime}, X_{2}^{\prime \prime}\right)$, starting from $X_{0}^{\prime \prime}=1$ which is coupled ex post facto with $\left(X_{0}, X_{1}, X_{2}\right)$ using the upward coupler $\mathcal{M}$. It is easily checked that $\mathbb{P}\left(X_{0} \in \cdot \mid X_{2}=X_{2}^{\prime \prime}=\mathbf{0}\right)=\nu_{0}(\cdot)$.

Observe that the initial distribution $\nu_{0}$ is stationary and so is the trajectory $X$, and that the kernel $k$ is time reversible. Fill's algorithm uses the following mechanism: The trajectory $\left(X_{0}, X_{1}, X_{2}\right)$ conditionally given $X_{2}=\mathbf{0}$ can be generated as if it started from $X_{2}=\mathbf{0}$ and ran backwards. Then it does not need to sample $X_{0}$ from $\nu_{0}$ but exhibits the distribution of $X_{0}$ exactly from $\nu_{0}$ if $X^{\prime \prime}$ is coalesced with $X$ by the time $n=2$ (i.e., $X_{2}=X_{2}^{\prime \prime}=\mathbf{0}$ ). In contrast, one can find $\mathbb{P}\left(X_{0} \in \cdot \mid X_{2}=X_{2}^{\prime}=\mathbf{0}\right)=\frac{3}{7} \delta_{\mathbf{0}}(\cdot)+\frac{4}{7} u_{2}(\cdot)$; thus, the algorithm would not work correctly if the trajectory $X^{\prime}$ were to be substituted for the trajectory $X^{\prime \prime}$.

\section{Existence of A BIVARIATE KERNEL AND SELECTIONS}

In what follows we consider a Polish space $R$ and the Borel $\sigma$-algebra $\mathcal{B}(R)$. Also by $\mathcal{M}_{1}(R)$ we denote the space of all probability measures on $R$. We will introduce a weak-* topology on $\mathcal{M}_{1}(R)$ by taking the open subbase of the form $\mathcal{O}_{G, \alpha}=\left\{p \in \mathcal{M}_{1}(R): p(G)>\alpha\right\}$ where $G$ is an open subset of $R$ and $\alpha \in \mathbb{R}$. This effectively makes $\mathcal{M}_{1}(R)$ a Polish space with the Prokhorov metric (cf. [2, 9]). Let $(\Omega, \mathcal{A})$ be a measurable space, and let $\Xi$ be a set-valued map on $\Omega$ whose values are closed nonempty subsets of $R$. The set-valued map $\Xi$ is said to be measurable if $\{\omega \in \Omega: \Xi(\omega) \cap G \neq \emptyset\} \in \mathcal{A}$ for every open subset $G$ of $R$. A map $\xi$ from $\Omega$ to $R$ is called a measurable selection of $\Xi$ if $\xi$ is measurable and $\xi(\omega) \in \Xi(\omega)$ for every $\omega \in \Omega$. The proof of the following selection theorem can be found in Aubin and Frankowska [1].

Theorem 2.1. If $\Xi$ is measurable, then there exists a measurable selection $\xi$ of $\Xi$.

Let $S$ be a Polish space equipped with a closed partial ordering $\leq$. Then the corresponding binary relation $\Delta \subseteq S \times S$ is closed (and is itself a Polish space). 
Moreover, the induced stochastic ordering $\preceq$ is closed (Kamae and Krengel [6]), and therefore, the binary relation $\mathcal{D}:=\left\{\left(p, p^{\prime}\right) \in \mathcal{M}_{1}(S) \times \mathcal{M}_{1}(S): p \preceq p^{\prime}\right\}$ is closed. We define a map $\Pi$ from $\lambda \in \mathcal{M}_{1}(\Delta)$ to $\left(p, p^{\prime}\right) \in \mathcal{D}$ by (1.1). Clearly $\Pi$ is continuous, and the Nachbin-Strassen theorem implies that it is surjective. The 'inverse' $\Pi^{-1}$ can be viewed as a set-valued map from $\mathcal{D}$ to the family of closed nonempty subsets of $\mathcal{M}_{1}(\Delta)$. A collection of probability measures in a Polish space is uniformly tight if and only if it is relatively compact in the weak-* topology (Prokhorov [9]). This observation is the key ingredient in the proof of the following lemma.

Lemma 2.2. $\Pi$ is a closed map, and $\Pi^{-1}$ is a measurable set-valued map.

Proof. Let $\mathcal{E}$ be a closed subset of $\mathcal{M}_{1}(\Delta)$. Suppose that $\left\{\left(p_{n}, p_{n}^{\prime}\right)\right\}_{n \geq 1} \subset \Pi(\mathcal{E})$ and $\left(p_{n}, p_{n}^{\prime}\right) \rightarrow\left(p, p^{\prime}\right)$. Then both $\left\{p_{n}\right\}_{n \geq 1}$ and $\left\{p_{n}^{\prime}\right\}_{n \geq 1}$ are relatively compact, and for any $\varepsilon>0$ there is a compact subset $K$ of $S$ such that $p_{n}(S \backslash K)<\varepsilon$ and $p_{n}^{\prime}(S \backslash K)<\varepsilon$ for all $n \geq 1$. For each $n \geq 1$ we can find $\lambda_{n} \in \mathcal{E}$ such that $\Pi\left(\lambda_{n}\right)=\left(p_{n}, p_{n}^{\prime}\right)$. Thus, we can define a compact subset $H=\pi_{1}^{-1}(K) \cap \pi_{2}^{-1}(K)$ for which $\lambda_{n}(\Delta \backslash H)<2 \varepsilon$ for all $n \geq 1$, and therefore $\left\{\lambda_{n}\right\}_{n \geq 1}$ is relatively compact. Let $\lambda$ be a cluster point for $\left\{\lambda_{n}\right\}_{n \geq 1}$, and without loss of generality, assume $\lambda_{n} \rightarrow \lambda$. Since $\lambda \in \mathcal{E}$ and $\Pi$ is continuous, $\left(p, p^{\prime}\right) \in \Pi(\mathcal{E})$. Hence, $\Pi$ is a closed map.

To show the measurability of $\Pi^{-1}$, let $\mathcal{O}$ be an open subset of $\mathcal{M}_{1}(\Delta)$. Since $\mathcal{M}_{1}(\Delta)$ is a metric space, we can find an increasing sequence $\left\{\mathcal{E}_{n}\right\}_{n \geq 1}$ of closed subsets such that $\bigcup_{n \geq 1} \mathcal{E}_{n}=\mathcal{O}$. Then $\left\{\left(p, p^{\prime}\right) \in \mathcal{D}: \Pi^{-1}\left(p, p^{\prime}\right) \cap \mathcal{O} \neq \emptyset\right\}=\Pi(\mathcal{O})=$ $\bigcup_{n \geq 1} \Pi\left(\mathcal{E}_{n}\right)$ is a Borel measurable subset of $\mathcal{D}$.

A Markov kernel $k$ on a Polish space $S$ can be viewed as a map from $r \in S$ to $k(r, \cdot) \in \mathcal{M}_{1}(S)$. It is an easy exercise to show that the measurability condition in the definition of the Markov kernel can be replaced by the requirement that $\left\{r \in S: k(r, \cdot) \in \mathcal{O}_{G, \alpha}\right\}$ be measurable for every open subbase $\mathcal{O}_{G, \alpha}$ of $\mathcal{M}_{1}(S)$. This, in turn, implies that $k$ is a Markov kernel if and only if it is a Borel measurable map from $S$ to $\mathcal{M}_{1}(S)$.

Theorem 2.3. Let $k$ and $k^{\prime}$ be Markov kernels on $S$. If $k$ is stochastically dominated by $k^{\prime}$, there exists a Markov kernel $K$ on $\Delta$ satisfying (1.3).

Proof. The pair $\left(k, k^{\prime}\right)$ is viewed as a measurable map from $\left(r, r^{\prime}\right) \in S \times S$ to $\left(k(r, \cdot), k^{\prime}\left(r^{\prime}, \cdot\right)\right) \in \mathcal{M}_{1}(S) \times \mathcal{M}_{1}(S)$. Then the map $\left(k, k^{\prime}\right)$ restricted to $\Delta$ is measurable, mapping from $\Delta$ to $\mathcal{D}$. By Theorem 2.1 and Lemma 2.2 there exists a measurable selection $\xi$ of $\Pi^{-1}$ from $\mathcal{D}$ to $\mathcal{M}_{1}(\Delta)$. Hence, we can construct a measurable map

$$
K\left(\left(r, r^{\prime}\right), \cdot\right)=\xi\left(k(r, \cdot), k^{\prime}\left(r^{\prime}, \cdot\right)\right)
$$

from $\Delta$ to $\mathcal{M}_{1}(\Delta)$ which satisfies (1.3).

A Markov kernel $k$ on $S$ is called (weak) Feller if $k(\cdot, G)$ is a lower semicontinuous function for every open subset $G$ of $S$ (cf. Meyn and Tweedie [8]). In the setting of Theorem 2.3 the Feller property is characterized by the continuity of the map $k$ from $S$ to $\mathcal{M}_{1}(S)$, which in turn implies that $\left(k, k^{\prime}\right)$ is a continuous map from $\Delta$ to $\mathcal{D}$. If $\Pi^{-1}$ admits a continuous selection, the bivariate kernel $K$ will inherit the Feller property.

The following counterexample shows, however, that such a selection does not always exist. Let $S=[0,2]$ and let $\left(s, s^{\prime}\right) \in \Delta$ if and only if $s=s^{\prime}$ or $\left(s, s^{\prime}\right) \in$ $[0,1] \times[1,2]$ satisfying either $s+1=s^{\prime}$ or $2-s=s^{\prime}$. It is easy to check that $\Delta$ is a closed partial order on $S$. 
Next, we introduce ternary fractions $t_{n i}=i 3^{-n}+3^{-n-1}, i=0,1, \ldots, 3^{n}-1$, on $[0,1]$, and define probability measures $v_{n}$ and $w_{n}$ on $\Delta$ by

$$
\begin{aligned}
& v_{n}(\cdot)=3^{-n} \sum_{i=0}^{3^{n}-1} \delta_{\left(t_{n i}, t_{n i}+1\right)}(\cdot), \\
& w_{n}(\cdot)=3^{-n} \sum_{i=0}^{3^{n}-1} \delta_{\left(t_{n i}, 2-t_{n i}\right)}(\cdot),
\end{aligned}
$$

for $n=1,2, \ldots$. Now both $\left(p_{n}, p_{n}^{\prime}\right)=\Pi\left(v_{n}\right)$ and $\left(q_{n}, q_{n}^{\prime}\right)=\Pi\left(w_{n}\right)$ converge to $\left(p, p^{\prime}\right)$ where $p$ and $p^{\prime}$ are Lebesgue measures (i.e., uniform distributions) supported by $[0,1]$ and $[1,2]$ respectively. However, if $\xi$ is any selection of $\Pi^{-1}$ as defined earlier, $\xi\left(p_{n}, p_{n}^{\prime}\right)=v_{n}$ and $\xi\left(q_{n}, q_{n}^{\prime}\right)=w_{n}$ converge to different points in $\mathcal{M}_{1}(\Delta)$. Hence, $\xi$ cannot be continuous.

\section{EXISTENCE OF AN UPWARD COUPLER}

A nonnegative function $\nu$ on $\Omega \times \mathcal{B}(S)$ is called a transition probability if $\nu(\omega, \cdot)$ is a measurable map from a measurable space $(\Omega, \mathcal{A})$ to a Polish space $\mathcal{M}_{1}(S)$ of probability measures.

Dynkin [3] proved the theorem below for the case of probability measures on $S$ (rather than transition probabilities).

Lemma 3.1. Let $\mu$ and $\nu$ be transition probabilities on $\Omega \times \mathcal{B}(S)$. If $\nu(\omega, \cdot)$ is absolutely continuous with respect to $\mu(\omega, \cdot)$ for each $\omega \in \Omega$, then there exists an $\mathcal{A} \otimes \mathcal{B}(S)$-measurable function $\gamma(\omega, s)$ such that

$$
\nu(\omega, E)=\int_{E} \gamma(\omega, s) \mu(\omega, d s), \quad E \in \mathcal{B}(S),
$$

for each $\omega \in \Omega$.

Proof. The Borel $\sigma$-algebra $\mathcal{B}(S)$ can be generated by an increasing sequence $\left\{\mathcal{C}_{n}\right\}_{n \geq 1}$ of $\sigma$-algebras, each of which consists of finitely many atoms. For every $n \geq 1$ we can define the $\mathcal{A} \otimes \mathcal{B}(S)$-measurable function $\gamma_{n}$ by

$$
\gamma_{n}(\omega, s)= \begin{cases}\nu(\omega, C) / \mu(\omega, C) & \text { if } \mu(\omega, C)>0 \\ 0 & \text { if } \mu(\omega, C)=0\end{cases}
$$

where $C \in \mathcal{C}_{n}$ is an atom such that $s \in C$. Let $\omega$ be fixed, and let $\rho_{\omega}$ be the RadonNikodym derivative of $\nu(\omega, \cdot)$ with respect to $\mu(\omega, \cdot)$. Observe that the sequence $\left\{\gamma_{n}(\omega, \cdot), \mathcal{C}_{n}\right\}_{n \geq 1}$ is a martingale and that $\int_{E} \gamma_{n}(\omega, s) \mu(\omega, d s)=\int_{E} \rho_{\omega}(s) \mu(\omega, d s)$ for each $E \in \mathcal{C}_{n}$ and $n \geq 1$. Thus, $\gamma_{n}(\omega, \cdot)$ converges to $\rho_{\omega}$ almost everywhere with respect to $\mu(\omega, \cdot)$ for every fixed $\omega$. Set $\gamma(\omega, s)=\lim _{\sup _{n \rightarrow \infty}} \gamma_{n}(\omega, s)$. Then it is $\mathcal{A} \otimes \mathcal{B}(S)$-measurable and satisfies (3.1).

Let $K$ be a Markov kernel on $\Delta$ satisfying (1.3), and let $B \in \mathcal{B}(S)$ be fixed. By Lemma 3.1 there exists a $\mathcal{B}(\Delta) \otimes \mathcal{B}(S)$-measurable function $M_{r, r^{\prime}}(s, B)$ such that

$$
K\left(\left(r, r^{\prime}\right), \pi_{1}^{-1}(E) \cap \pi_{2}^{-1}(B)\right)=\int_{E} M_{r, r^{\prime}}(s, B) k(r, d s), \quad E \in \mathcal{B}(S),
$$

for each $\left(r, r^{\prime}\right) \in \Delta$. 
Viewed as a set function on $\mathcal{B}(S), M_{r, r^{\prime}}(s, \cdot)$ may not necessarily be a probability measure. Thus, $M_{r, r^{\prime}}(s, B)$ must be modified to obtain a probability measure $\tilde{M}_{r, r^{\prime}}(s, \cdot)$ for which (3.2) is still valid. We adopt the standard constructive argument for existence of regular conditional probability (cf. Dudley [2]) to prove the following.

Theorem 3.2. There exists a version of $M_{r, r^{\prime}}(s, \cdot)$ which is a probability measure satisfying (3.2).

Proof. Recall that $S$ is a Polish space of cardinality of the continuum and is therefore Borel isomorphic to $[0,1]$ (see [2]). Thus, it suffices to show the claim for $S=[0,1]$. Consider the algebra $\mathcal{U}$ generated by a countable open base in $S$. Note that each $U \in \mathcal{U}$ is expressed as a countable union of closed subsets. Since $S=[0,1]$ is compact, we can choose a specific sequence of increasing compact subsets $F_{n}$ 's such that

$$
\lim _{n \rightarrow \infty} F_{n}=U .
$$

Let $\mathcal{V}$ be the algebra generated by $\mathcal{U}$ and the collection of all such sequences $\left\{F_{n}\right\}_{n=1}^{\infty}$. Fix $\left(r, r^{\prime}\right) \in \Delta$. Then the collection of $M_{r, r^{\prime}}(s, \cdot)$ satisfies the following properties for almost every $s \in S$ with respect to $k(r, \cdot)$ :

(i) $M_{r, r^{\prime}}(s, V) \geq 0$ for $V \in \mathcal{V}$;

(ii) $M_{r, r^{\prime}}(s, \emptyset)=0$ and $M_{r, r^{\prime}}(s, S)=1$;

(iii) $M_{r, r^{\prime}}\left(s, \bigcup_{i=1}^{n} V_{i}\right)=\sum_{i=1}^{n} M_{r, r^{\prime}}\left(s, V_{i}\right)$ if $V_{1}, \ldots, V_{n} \in \mathcal{V}$ are disjoint;

(iv) $\lim _{n \rightarrow \infty} M_{r, r^{\prime}}\left(s, F_{n}\right)=M_{r, r^{\prime}}(s, U)$ where $\left\{F_{n}\right\}_{n=1}^{\infty}$ is the sequence in (3.3).

Define $W$ to be the set of all $\left(r, r^{\prime}, s\right)$ in $\Delta \times S$ for which (i)-(iv) above hold. Since $W$ is defined by countably many equations, it is a measurable subset of $\Delta \times S$. Furthermore, the projection $W_{r, r^{\prime}}:=\left\{s \in S:\left(r, r^{\prime}, s\right) \in W\right\}$ on $S$ satisfies $k\left(r, W_{r, r^{\prime}}\right)=1$ for each $\left(r, r^{\prime}\right) \in \Delta$. Fix a $\left(r, r^{\prime}, s\right) \in W$. Then (iv) implies that $M_{r, r^{\prime}}(s, \cdot)$ is regular on $\mathcal{U}$ for $\mathcal{V}$ (see Section 10.2 of [2]), which implies it is countably additive on $\mathcal{U}$ and can be extended to a probability measure $\tilde{M}_{r, r^{\prime}}(s, \cdot)$ on $\mathcal{B}(S)$. Set $\tilde{M}_{r, r^{\prime}}(s, \cdot)=\delta_{s}(\cdot)$ for $\left(r, r^{\prime}, s\right) \notin W$, thus obtaining a collection of probability measures $\tilde{M}_{r, r^{\prime}}(s, \cdot)$ for $\left(r, r^{\prime}, s\right) \in \Delta \times S$. This $\tilde{M}_{r, r^{\prime}}(s, \cdot)$ will be a version of $M_{r, r^{\prime}}(s, \cdot)$ satisfying (3.2) by the following monotone class argument. Define $\mathcal{E}$ to be the class of all subsets $B \in \mathcal{B}(S)$ such that $\tilde{M}_{r, r^{\prime}}(s, B)$ is $\mathcal{B}(\Delta) \otimes \mathcal{B}(S)$-measurable and satisfies (3.2) for all $\left(r, r^{\prime}\right) \in \Delta$. Using the properties of $W_{r, r^{\prime}}$ outlined above, it is easy to see that $\mathcal{E}$ is a monotone class that contains $\mathcal{U}$ and thus must be equal to $\mathcal{B}(S)$.

Using Theorems 2.3 and 3.2 we can assume without loss of generality that $M_{r, r^{\prime}}(s, \cdot)$ satisfies (1.4). Let $\mathcal{G}$ be a countable open base of $S$, and let $D_{U}=$ $\left\{s \in S: s \leq s^{\prime}\right.$ for some $\left.s^{\prime} \in U\right\}$ be the down set generated by $U \in \mathcal{G}$. Then the probability measure $M_{r, r^{\prime}}(s, \cdot)$ satisfies

$$
M_{r, r^{\prime}}(s, U)=0, s \notin D_{U} \text { for } U \in \mathcal{G},
$$

almost everywhere with respect to $k(r, \cdot)$. Now (3.4) lists countably many equations. Therefore, just as in the proof of Theorem 3.2 above, we can replace $M_{r, r^{\prime}}(s, \cdot)$ with $\delta_{s}(\cdot)$ for some $\left(r, r^{\prime}, s\right)$ as necessary, so that (3.4) holds for all $s \notin D_{U}$ and (3.2) holds for every $B \in \mathcal{B}(S)$. Hence $M_{r, r^{\prime}}(s, U)=0$ whenever $U \in \mathcal{G}$ and $U \cap\left\{s^{\prime}: s \leq s^{\prime}\right\}=\emptyset$ 
for each $\left(r, r^{\prime}, s\right)$. Thus, the support of $M_{r, r^{\prime}}(s, \cdot)$ is contained in $\left\{s^{\prime}: s \leq s^{\prime}\right\}$, which leads to the following statement.

Theorem 3.3. If $k$ is stochastically dominated by $k^{\prime}$, then there exists an upward coupler $\mathcal{M}$ satisfying (1.4).

\section{ACKNOWLEDGMENT}

The authors would like to thank the referees for a number of valuable suggestions that helped to improve the exposition and clarify a number of results.

\section{REFERENCES}

1. Aubin, J.-P., and Frankowska, H. (1990). Set-Valued Analysis. Birkhäuser, Berlin. MR 1048347 (91d:49001)

2. Dudley, R. M. (1989). Real Analysis and Probability. Wadsworth, Inc., Pacific Grove, CA. MR.982264 (91g:60001)

3. Dynkin, E. B. (2006). On extreme X-harmonic functions. Math. Res. Lett. 13, 59-69. MR 2199566 (2007d:60045)

4. Fill, J. A., Machida, M., Murdoch, D. J., and Rosenthal, J. S. (2000). Extension of Fill's perfect rejection sampling algorithm to general chains. Random Structures and Algorithms 17, 290-316. MR.1801136 (2001m:60164)

5. Kamae, T., Krengel, U., and O'Brien, G. L. (1977). Stochastic inequalities on partially ordered state spaces. Ann. Probab. 5, 899-912. MR0494447 (58:13308)

6. Kamae, T., and Krengel, U. (1978). Stochastic partial ordering. Ann. Probab. 6, 1044-1049. MR.512419 (80b:60020)

7. Nachbin, L. (1965). Topology and Order. Van Nostrand, New York. MR0219042 (36:2125)

8. Meyn, S. P., and Tweedie, R. L. (2009). Markov Chains and Stochastic Stability. Second edition. Cambridge University Press, Cambridge. MR 2509253

9. Prokhorov, Yu. V. (1956). Convergence of random processes and limit theorems in probability theory. Theor. Probability Appls. 1, 157-214. MR0084896 (18:943b)

10. Strassen, V. (1965). The existence of probability measures with given marginals. Ann. Math. Statist. 36, 423-439. MR0177430 (31:1693)

Department of Mathematics, Tennessee Technological University, Cookeville, Tennessee 38505

E-mail address: mmachida@tntech.edu

Department of Mathematics, Tennessee Technological University, Cookeville, Tennessee 38505

E-mail address: alex@math.tntech.edu 